\title{
Reviewing the value of leukocytes cell population data (CPD) in the management of sepsis
}

\author{
Eloísa Urrechaga \\ Biocruces Bizkaia Health Research Institute, Cruces Plaza, Bizkaia, Spain \\ Correspondence to: Eloísa Urrechaga. Biocruces Bizkaia Health Research Institute, Cruces Plaza, 48903 Barakaldo, Bizkaia, Spain. \\ Email: eloisa.urrechagaigartua@osakidetza.net.
}

\begin{abstract}
Sepsis is a medical emergency that describes the body's systemic immune response to an infection and can lead to end-stage organic dysfunction and death. Despite the advances in understanding the pathophysiology of this syndrome and therapies, sepsis remains one of the leading causes of morbidity and mortality in critically ill patients. Early diagnosis and rapid intervention are essential to improve outcomes, which inspired the concept "golden hour," during which the correction of shock and organic dysfunction can improve the patients' outcomes. But the initial presentation of sepsis is often nonspecific and its severity is difficult to assess. Anomalies in temperature, heart and respiratory rates and leukocyte counts are manifestations of systemic inflammatory response syndrome (SIRS). Diagnosis, management and followup of patients with sepsis remains a challenge, and diverse biomarkers have been proposed for the timely diagnosis and prognosis of septic patients: lactic acid, procalcitonin (PCT), C-reactive protein, immature granulocytes. The host's initial response to infection is a humoral, cellular and neuroendocrine reaction to infection, and leukocytes interact with endothelial cells. The new generation of hematological analyzers incorporates technological innovations allowing to expand the information derived from the complete blood count: new leukocyte derived parameters are emerging as potentially useful markers in different clinical situations. Additional research parameters cell population data (CPD), characterizing different leukocyte populations have become available, and preliminary observations suggest their utility in the diagnosis of sepsis. This review emphasizes the value of CPD, reported by modern cellular counters for early recognition of sepsis, and therefore the potential improvement in patient outcomes.
\end{abstract}

Keywords: Sepsis; leukocytes; cell population data (CPD).

Submitted Oct 15, 2019. Accepted for publication Jul 10, 2020.

doi: $10.21037 /$ atm-19-3173

View this article at: http://dx.doi.org/10.21037/atm-19-3173

\section{Recognizing sepsis as a global health priority}

Sepsis is one of the key problems of modern medicine due to its widespread prevalence in the world and high mortality. Despite significant advancements in the understanding of its pathophysiology and advances in hemodynamic monitoring, sepsis remains one of the major causes of morbidity and mortality in critically ill patients (1).

The global epidemiological burden of sepsis is, however, difficult to be accurately calculated. The estimation accounts for more than 30 million people affected by sepsis annually worldwide, resulting in 6 million deaths (2).
But the likelihood that the result is a significant underestimate is evident, because calculations are based on data on in-patients from high-income countries. The hospital mortality of patients with sepsis in North America and Europe ranges from $28.3 \%$ to $41.1 \%$ (3). But $87 \%$ of the world's population lives in low- and middle-income countries, and data from these areas are lacking.

Early diagnosis and rapid intervention have been directly related to improved outcomes for trauma patients, which inspired the concept of the "golden hour". Every hour of delay of the therapy start results in an increase in sepsisrelated mortality by about $7-10 \%$ (4). 
The time-sensitive aspect of this disease makes crucial early recognition and treatment of infection. But around $70 \%$ of sepsis cases are community-acquired, making sepsis a problem not only in the hospital setting; general practitioners must be aware to manage the disease early enough to prevent delayed treatment, which is the cause of disability and death (4).

\section{Innate inflammation: the role of neutrophils and monocytes}

The major problem for patients at risk for sepsis is an immunological imbalance.

Cells of the innate immune system, such as monocytes and neutrophils, are the first-line of defense against infections. In the presence of sepsis, these cells produce inflammatory cytokines, causing widespread inflammation that can lead to death.

Neutrophils play the key role in protecting against microbial infections, which is due to the presence of a large number of proteolytic enzymes and rapid production of reactive oxygen species to reduce pathogens. If these lytic factors and pro-inflammatory cytokines are released extracellularly from the neutrophil infiltrated tissue, this results in the local lesion. In sepsis, a local infection is accompanied by systemic activation of neutrophils.

Elimination of neutrophils through apoptosis is a homeostatic mechanism that prevents damage to healthy tissues. This process is key to the prevention and resolution of inflammation. Apoptosis of neutrophils is suppressed in patients with systemic inflammation, systemic infections, sepsis and in those at risk of multiple organ dysfunction, which is due to the activity of circulating lipopolysaccharide, lipoteichoic acid and pro-inflammatory cytokines (5).

Stimulated polymorphonuclear leukocytes bind to adhesion molecules on the surface of endothelial cells in organs; in those binding sites, they secrete proteolytic enzymes and oxygen metabolites which leads to endothelium damage and endothelial barrier disruption, increased capillary permeability, and infiltration of inflammatory mediators of the parenchyma of organs. Thus, the function of neutrophils is to eliminate foreign bacteria, but under certain circumstances their activity can lead to organ failure (6-8).

Monocytes are involved in immune response from very early stages, as first-line defense cells against infection. Monocytes perform multiple immune functions including phagocytosis, antigen presentation and cytokine production.
Their functions are regulated by cell surface receptors: CD14, the receptor of lipopolysaccharides produced by bacteria and serum proteins; receptors such as CD163 that removes components of damaged cells (9).

Monocytes are a heterogeneous set of cells which differ in phenotype, size, nuclear morphology and function. In sepsis, this variety is even more pronounced due to functional changes in certain monocyte subsets, and is reflected in a variation in the morphology of monocytes (10). The monocytes' function in sepsis pathogenesis are diverse, related to the decreased antigen presentation capacity and decreased pro-inflammatory cytokine release. Their key role in disease progression involves the possible polarization from a pro-inflammatory state to an immunosuppressive state $(9,10)$.

\section{Innovative technologies in the evaluation of the leucocytes functional activity}

Over the past few decades, hematology analyzers have undergone rapid development due to the technological progress. This is evident not only in the advances in full automation and better performance, also the number of parameters reported by these analyzers has increased.

The new technology is able to quickly examine a large number of cells to provide complete blood cell profiles. Hematology analyzers record electronic signals that evaluate morphology and cellular characteristics. The use of algorithms and sophisticated software transforms this raw data into reportable results of cell counts and derived research parameters (11).

This improved technology allows new parameters to be reported along with the basic full blood count (CBC). The cell population data (CPD) provides quantitative information on the morphological and functional characteristics of leukocytes (neutrophils, monocytes and lymphocytes). The morphologic characteristics can be accurately measured; more detailed study of these cells and their morphological variations in response to stimuli, such as infections, can offer valuable information on the state of activation and functional activity.

The clinical application of CPD parameters offers several advantages. These are parameters generated during CBC without additional sample needs, available 24/7. Being numbers, CPD are, more objective and accurate than manual differential counts, because thousands of leukocytes (WBC) are automatically evaluated $(12,13)$. CBC is routinely requested by clinicians as part of the management 
of patients with sepsis. Therefore, the CPD could be used as an additional marker at a lower cost than other laboratory tests.

\section{Coulter technology}

Leukocyte morphological parameter were originally used by service engineers to verify the optimal setup for each of the four main leukocyte subpopulations in the Coulter hematology analyzers (Beckman Coulter Inc., Miami, FL, USA). This manufacturer applies the VCS technology to evaluate the morphology of leukocytes in their 'near native states' using three independent energy sources simultaneously.

$\mathrm{V}$ direct current impedance measures cell volume, accurate size of all cell types and the degree of cell size variation are reported; $\mathrm{C}$ radio frequency opacity characterizes conductivity, a measurement of the internal composition of each cell; S laser beam measures light scatter, related to cytoplasmic granularity and nuclear structure.

The "Coulter method" measures the changes in electrical resistance when the cell, suspended in a conductive diluent, passes through a small aperture called the sensing zone. Two electrodes, one located on each side of the aperture, produce a resistance; as each cell passes through this area the electrical path momentarily increases the resistance, generating an electrical pulse. The accumulation of pulses is processed for coincidence correction, and multiplied by a calibration factor, rendering the WBC counts.

The Coulter counter LH series give CPD for neutrophils, lymphocytes, monocytes, and eosinophils with their mean values and standard deviations (SD). For example, for neutrophils, these data which are given include MNV (mean neutrophil volume), MNV-SD, volume distribution width (VDW) (SD of neutrophil volume), MNC (mean neutrophil conductivity), MNC-SD, CDW (SD of neutrophil conductivity), MNS (mean neutrophil scatter), and MNS-SD (SD of neutrophil scatter).

Similar parameters apply to lymphocytes, monocytes, and eosinophils (14-16).

In addition to volume and conductivity measurements, a new flow cell design in the newest model of Coulter UniCel DxH 800 (Beckman Coulter, Miami, FL, USA) analyzer supports multiple angles of light scatter measurements enabling enhanced data acquisition for the leukocyte differentials.

Combining the additional light scatter measurements
UniCel DxH 800 Coulter can improve the differential accuracy and flagging efficiencies (17-19). CPD values are reported as arbitrary units of light scatter, au, as previously described (19).

There are 3 additional measurements of the scatter compared to that in previous Coulter models such as the LH750. Median angle light scatter (MALS), lower MALS (LMALS), and upper MALS (UMALS) provide information concerning granularity and the membrane surface, axial light loss measurement of cellular transparency, and the low angle light scatter (LALS) cellular complexity index, respectively.

\section{Fluorescence flow cytometry (Sysmex)}

Another company, Sysmex (Sysmex Corporation, Kobe, Japan), can now report CPD, based in different principles. The technology applied is based on fluorescence-flow cytometry using blood-cell membrane surfactant reagents, and different fluorescence dyes specific for staining nucleic acids and proteins. The generation of bioactive lipids from cell-membranes and the measurement of the scatter-light render information about shape, size and inner granularity of cells $(20,21)$.

A hematology counter applying this technology and automated-gating is potentially useful for detecting the activation of neutrophils and monocytes in real-time and in an accurate and reproducible way (20). In the XN analyzers, the leukocyte differential channel discriminates the leukocytes and the signals are plotted in a scattergram (WDF).

The optical signals along $\mathrm{X}$-axis (side scatter) are proportional to the internal complexity; fluorescence along Y-axis correlates with the nucleic acid content, while forward scatter (Z-axis) is related to cell size. Means and SD of values are recorded for each leukocyte subset. The width of dispersion of the values represents heterogeneous signals. Those parameters, calculated according to distribution width, represents the distribution range of the major cellular population, excluding outliers below $20 \%$ peak height in the distribution curve.

CPD generated by Sysmex XN analyzers has been well summarized in a previous work (22). The following CPD parameters are reported along the $\mathrm{X}$-axis: neutrophils complexity (NE-SSC), lymphocytes complexity (LY-X), monocytes complexity (MO-X), width of dispersion of neutrophils complexity (NE-WX), width of dispersion of lymphocytes complexity (LY-WX) and width of dispersion of monocytes complexity (MO-WX). 
$\mathrm{CPD}$ on the Y-axis: neutrophils fluorescence intensity (NE-SFL), lymphocytes fluorescence intensity (LY$\mathrm{Y})$, monocytes fluorescence intensity (MO-Y), width of dispersion of neutrophils fluorescence (NE-WY), width of dispersion of lymphocytes fluorescence (LY-WY) and width of dispersion of monocytes fluorescence (MOWY).CPD reported on the $Z$-axis: neutrophils size (NEFSC), lymphocytes size (LY-Z), monocytes size (MO-Z), width of dispersion of neutrophils size (NE-WZ), width of dispersion of lymphocytes size (LY-WZ) and the width of dispersion of monocytes size (MO-WZ).

\section{CPD in sepsis}

The CPD were first reported by Coulter automated hematology analyzers, in those days LH750. Being the first company to make CPD available to laboratory professionals, the clinical usefulness of CPD by Coulter hematology analyzers is the most studied.

The diagnostic utility of neutrophil CPD in acute bacterial infection, particularly roles in the differentiation between post-surgical infections and SIRS. CPD show also in viral infection triggers specific changes on lymphocyte morphology and thus the CPD values changes accordingly, providing a potential new hematological parameter for viral infection. Monocyte CPD are useful in various Infections discriminating malaria and dengue infections from other febrile maladies, also changing distinctively in acute hepatitis B viral infection (15).

Few studies have used CPD values in neonates, and all concerned in diagnosing neonatal sepsis. Raimondi et al. (23) applied neutrophil volume for the screening of late-onset sepsis in low birthweight neonates. They reported that at a cutoff of 148 , the sensitivity, specificity, positive predictive value (PPV) and negative predictive value (NPV) were 95\%, $88 \%, 61.3 \%$ and $98.9 \%$, respectively for MNV. For VDW, at a cutoff value of 27.5 , sensitivity, specificity, PPV and NPV were $80 \%, 52 \%, 25 \%$ and $92 \%$, respectively.

The combination of both $\mathrm{MNV}$ and $\mathrm{C}$ reactive protein (CRP) had a sensitivity of $95 \%$, specificity of $97 \%$, PPV of $86.4 \%$ and NPV of $99 \%$.

Lee et al. (24) reported that MNC <145.6 and MNC-SD $>10.4$ from cord blood were the predictive for the diagnosis of early neonatal sepsis in neonates with chorioamnionitis.

Celik et al. $(16,25)$ evaluated the volume, conductivity, and light scatter of neutrophils in diagnosing sepsis, and found them were reliable for screening and for the management of treatment. They suggested to use a combination of CRP, the mean and SD of neutrophil volume, and interleukin-6 (IL-6) levels for screening purpose.

The optimal cutoff levels of $M N V>157.15$; VDW $>37.44$; $\mathrm{MNC}<159.3$; CDW $>12.3$; MNS $<127.5$.

MNV sensitivity $79 \%$, specificity $82 \%$, PPV $90 \%$, and NPV 65\%; VDW sensitivity $60 \%$, specificity $78 \%$, PPV $85 \%$, and NPV $48 \%$. The combination of IL-6, CRP, and MNV rendered sensitivity $94 \%$, specificity $88 \%$, PPV $95 \%$ and NPV $86 \%$ for diagnosis of sepsis. This combination had higher sensitivity and NPV than those of each test alone (16).

When VCS parameters were evaluated to diagnose sepsis in adult population, CPD related to neutrophils, $\mathrm{MNV}$, and VDW and MNS, were found to be useful.

Chaves et al. (14) evaluated the Coulter parameters in adults with proven sepsis.

A significant increase in $\mathrm{MNV}$ of septic patients was found when compared to controls $(156 \pm 13.5$ vs. $143 \pm 4.8$; $\mathrm{P}<0.001)$.

The increase in MNV was associated with higher WBC counts and percentage of neutrophils; this pattern was present even in patients who did not have leukocytosis or neutrophilia. At a cutoff of 150 , sensitivity $70 \%$ and specificity $91 \%$ were achieved (14).

Cortegiani et al. (26) reported an AUC for MNV 0.816 (95\% CI, 0.722 to 0.889 ); the optimal cut-off was 163 rendered a sensitivity of $84.0 \%$ and specificity $68.75 \%$.

The number of immature neutrophils increases during acute bacterial infection. However, the determination of band counts and other morphologic changes in the cytoplasm (e.g., toxic granulation and vacuolization, Döhle bodies), is time consuming, and requires an experienced professional. By this means, Chaves et al. (27) evaluated Neutrophil VDW, NDW. The septic patients showed a significant increase in the NDW $(24.7 \pm 4.5)$ compared with the controls $(19.0 \pm 1.5 ; \mathrm{P}<0.001)$.

The high values were observed even in patients with white blood cell counts $<11 \times 10^{9} / \mathrm{L}$. At a cut off 23 , sensitivity of $69 \%$ and specificity of $100 \%$ were obtained.

The conclusion was that NDW is a quantitative parameter reliable as additional indicator of acute infection.

Mardi et al. (28) studied neutrophil and monocyte VCS parameters in adults, reporting significant increase in MNV and $M M V$ in the sepsis group when compared to non -systemic infections and controls. At a cut-off of $150 \mathrm{MNV}$ had a comparable sensitivity and specificity as CRP (cutoff point $60 \mathrm{mg} / \mathrm{dL}$ ), and was the most predictive CPD. 
Taken together MNV and MMV, have potential value to discriminate sepsis and limited infections.

The UniCel DxH 800 was launched after LH series. Park et al. (19) evaluated the clinical usefulness of neutrophil CPD, as determined using the Coulter DxH 800 counter, for screening sepsis. The results in sepsis samples were the significantly high volume of neutrophils and monocytes and the significantly low light scattering of neutrophils. This study also reported the performance of lymphocyte SD parameters sensitivity and specificity ranging $78-87 \%$ ), monocyte volume (at cut off 177.5 sensitivity of $88.2 \%$; specificity of $87.3 \%)$.

Monocyte volume SD resulted to have diagnostic performances (sensitivity $93.1 \%$; specificity $91.0 \%$, cut off 22.16) comparable to those of immature granulocytes, IG (sensitivity $82.8 \%$; specificity $90.8 \%$ ).

These data were confirmed by Lee et al. (29), in a population of elderly patients. They found that MNV at a cut-off 156.5 , showed a sensitivity of $83.3 \%$ and specificity of $78 \%$ in the prediction of sepsis.

Mammen et al. (30) probed the potential value of both neutrophil and monocyte derived CPDs; in their cohort MNV was significantly higher $(\mathrm{P}<0.001)$ in patients with sepsis $(165.5 ; 95 \% \mathrm{CI}, 161.6-169.4)$ than in nonseptic (157.3; 95\% CI, 154.6-160.1) patients and donors (148.9; 95\% CI, 147.9-150). A similar pattern was seen for mean monocyte volume MMV. The AUC was highest for MMV (0.74), MNV and MMV had the highest specificity of $85 \%$ and $80 \%$, respectively.

These results were confirmed by Arora et al. (31). MNV and $M M V$ were found to be highly informative. A cutoff value of 150.2 for MNV gave a sensitivity and specificity of $79.1 \%$ and $95 \%$, respectively, with an area under the curve (AUC) of $92.3 \%$. With a cutoff of 168.3, MMV had a sensitivity of $80.6 \%$ and specificity of $77.5 \%$, AUC of $83 \%$.

Recently, the Monocyte Distribution Width MDW has been approved by the Food and Drug Administration (FDA) to improve the early detection of sepsis (32). Compared with other parameters, MDW was the best for discriminating sepsis and all other conditions (AUC, 0.79; at cut off 20.5 sensitivity $77 \%$; specificity $73 \%$ ); sepsis from SIRS (AUC 0.74), and severe sepsis from non-infected patients (AUC 0.88; NPV 99\%).

The added value of MDW was statistically significant (AUC, 0.89 for $M D W+W B C$ vs. 0.81 for WBC only; $\mathrm{P}<0.01$. Using both MDW and WBC count improves the detection of sepsis at the time of admission, compared with WBC alone.
The clinical relevance of CPD as complementary biomarkers for the diagnosis of sepsis, as measured using Sysmex XE-5000 (Sysmex, Kobe, Japan) was evaluated by Luo et al. in 2013 (33).

They evaluated CPD related to the cytoplasmic structures of neutrophils (Neut-X), cellular nucleic acid content (Neut-Y), and Neut-Z; this is calculated using the equation:

Neut- $Z=\left(\text { Neut }-X^{2}+\text { Neut }-Y^{2}\right)^{1 / 2}$, in the two $X Y$ axis of the WDF scattergram.

CRP, Neut-X, Neut-Y and Neut- $Z$ values were found to be significantly higher in the septic patients. In addition, Neut-X, Neut-Y, and Neut-Z correlated with CRP and PCT values. Good results were obtained in the ROC analysis for the diagnosis of sepsis, AUC $>0.80$.

NE-SFL and NE-WY, reported by Sysmex XN analyzer, were found to be a reliable aid in detection of sepsis, by Park et al. (34). AUC (0.909 and 0.905, respectively) were comparative or higher than those obtained for routine $\mathrm{CBC}$ tests.

The correlation with the severity of disease was explored by Buoro et al. (35). The values of neutrophils fluorescence intensity and monocytes internal structure (NE-SFL and $\mathrm{MO}-\mathrm{X}$ ) were higher in septic shock compared to patients who do not develop sepsis. Overall, MO-X and NESFL exhibited the best performance for diagnosing sepsis in all patients (AUC, 0.75 and 0.72). These parameters significantly correlated with SOFA score suggesting that NE-SFL and MO-X may provide useful information for the management of septic patients.

Shalini et al. (36) enrolled 100 healthy subjects and 100 clinically suspected cases of sepsis with quick sequential organ failure assessment (qSOFA) score $>2$.

Except for neutrophil, monocyte cell size (NE-FSC, $\mathrm{MO}-\mathrm{Z})$ and lymphocyte fluorescence intensity (LY-Y) all other CPD parameters show significant difference $(\mathrm{P}<0.001)$ in sepsis group compared to healthy controls. LY-Y parameter showed a negative correlation in septic patients, which is consistent with Buoro et al. reports (35).

The importance of NE-SFL and MO-X in sepsis cases was also confirmed by Urrechaga et al. (37). It is emphasized in a new proposed scale: NEMO (neutrophils and monocytes) is a scoring system for early diagnosis of sepsis based on these parameters. It includes diverse CBC and CPD tests: neutrophil count, lymphocyte count, IG, NE-SFL and MO-X.

Based on those parameters a score is composed, and a risk stratification scale for diagnosing sepsis was 
established. The NEMO score classifies patients based on the probability of develop sepsis, according to the values NEMO 0-3 (low probability), 4-5 (medium probability) and $\geq 6$ (high probability).

The management of critically ill patients is often challenging for clinicians, and the prediction of infection and its severity remains difficult. The scoring systems, Sequential Organ Failure Assessment (SOFA) and/or Acute Physiology and Chronic Health Evaluation II (APACHE II) are useful tools in daily practice. Based in these well-known scores, the intensive care infection score (ICIS), derived from five blood-cell parameters that characterize the innate immune response in routine samples could be helpful in this respect. The five parameters reported by Sysmex analyzers are: fluorescence intensity neutrophils, hemoglobin content of reticulocytes, absolute neutrophils count, antibody secreting lymphocytes and absolute immature granulocytes count. In parallel to CRP and PCT, ICIS score is increases in critically ill patients with probable infection.

The advance is that data for calculation can be collected routinely from the same CBC blood sample and at lower cost if compared to CRP and PCT $(38,39)$, making ICISs a potentially useful predictor of infection and sepsis in patients in the ICU.

The CPD found to be useful in the detection of sepsis are shown in Table 1.

\section{CPD: opportunity and weakness}

In addition to above mentioned clinical utility in detecting sepsis, there are also other practical advantages. These parameters are generated during automated differential analysis without additional specimen requirements. They are quantitative, more objective and more accurate than manual differential counts, as previously stated.

Moreover, CPD offer robust turn-around-time and are more cost-effective. Since the hematology analyzers could determine not only leukocytes counts, but also evaluate the morphologic features of these leukocytes, the diagnostic accuracy improves, combining both leukocyte numerical and morphological parameters to differentiate the activation of cells.

The leukocyte CPD have much smaller overall withinsubject and between-subject biological variations compared to $\mathrm{WBC}$ differentials, suggesting that these morphological parameters are less variable around the homeostatic set point intra- and inter-individually, a low index of individuality (40).
The reference change value (RCV) for leukocyte $\mathrm{CPD}$ has also been documented (17).

The RCV or critical difference is defined as the percentage change that should be exceeded to verify a significant difference between two consecutive measurements.

It is calculated based on the analytical and biological variations of every laboratory test. The RCV can be applied not only. In the evaluation of patient's serial results, in special for test with a low index of individuality (as CPD), but also to generate objective delta-check values for use in quality control $(41,42)$.

As mentioned, CPDs represent raw measurements which depend on the optical design of the analyzer employed. Reliance on an individual instrument can be a serious limitation to the widespread use of the CPD. Due to the lack of standardization, these parameters cannot be used interchangeably and instrument-specific clinical decision limits are required (11). Along the lack of harmonization among different instruments and clinical laboratories, another disadvantage of these CPD parameters is less analytical quality specification. Metrological issues, such as stability, quality control targets and harmonization of CPD, are now under intense evaluation $(43,44)$.

\section{The laboratory in the management of sepsis}

Sepsis is a complex inflammatory syndrome with high prevalence among hospitalized patients, and represents high risk of mortality. Traditionally, the diagnosis was based on the presence of two or more positive SIRS criteria due to infection. However, the recently published sepsis-3 criteria place more emphasis on organic dysfunction caused by infection in the definition of sepsis (45). Nevertheless, there is no gold standard for diagnosis, and clinicians still rely on a number of biomarkers (standard or new) to discriminate between patients with and without infection as a cause of deterioration $(46,47)$.

The laboratory in the management of sepsis comprises key challenges. Early diagnosis is critical because an early treatment can reduce mortality. On the other hand, biomarkers with high NPV can aid to rule out sepsis and thus to reduce the use of unnecessary antibiotics. Other characteristics of an ideal biomarker for sepsis detection include the discrimination of systemic inflammation from infection, and bacterial from other infection, giving insights on etiology.

PCT is currently an FDA approved test to aid in the diagnosis of sepsis, but its performance is questionable. 
Table 1 Summary of the CPD found useful for detecting sepsis with the optimal cutoffs (arbitrary optical units) reported in literature

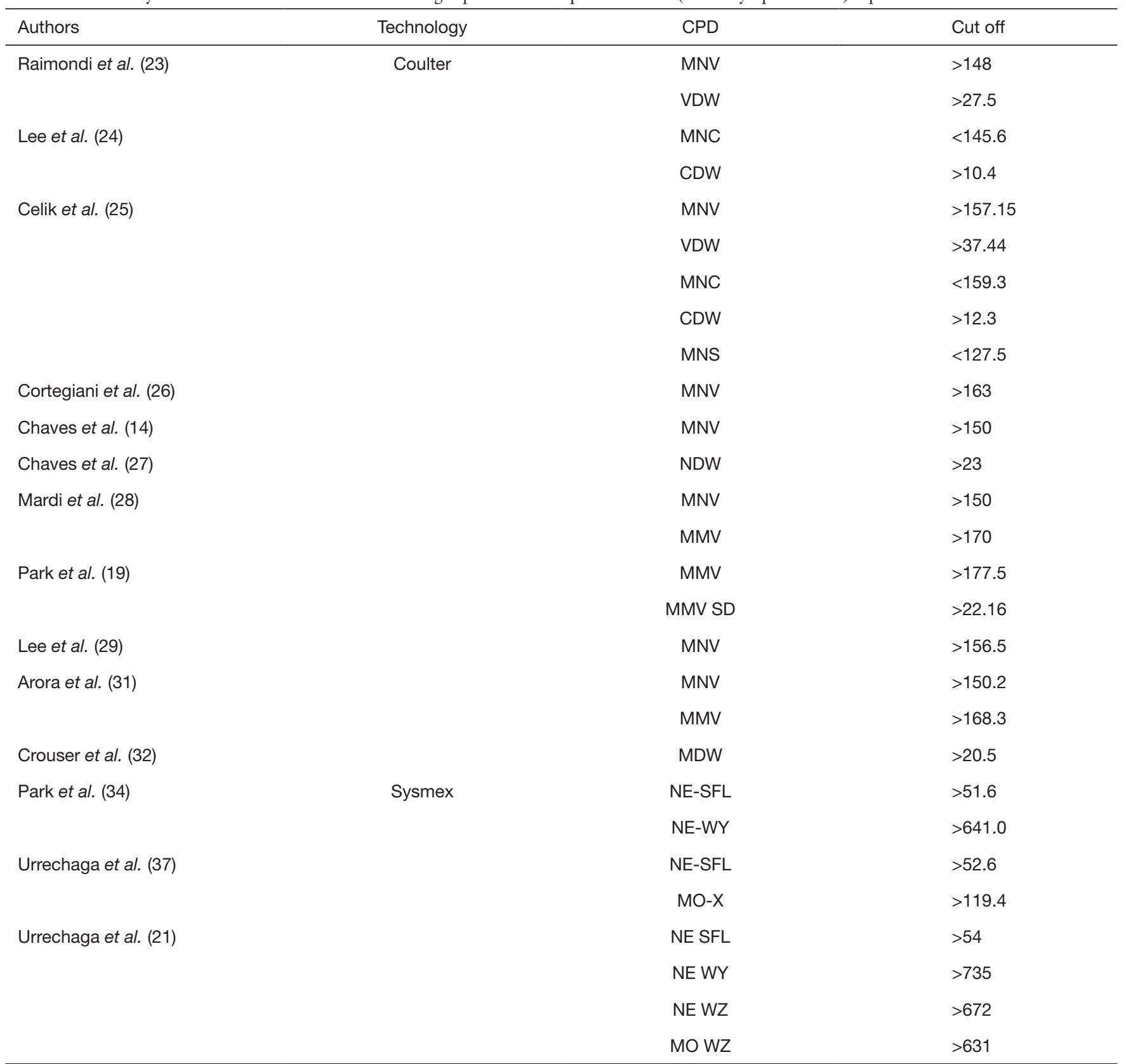

MNV, mean neutrophil volume; VDW, volume distribution width; NDW, standard deviation of neutrophil volume; MNC, mean neutrophil conductivity; CDW, standard deviation of neutrophil conductivity; MNS, mean neutrophil scatter; MMV, mean monocyte volume; MMV SD, standard deviation of monocyte volume; MDW, monocyte volume distribution width; NE-SFL, neutrophils fluorescence intensity; NE-WY, width of dispersion of neutrophils fluorescence; MO-X, monocytes complexity; NE-WZ, width of dispersion of neutrophils size; MO-WZ, width of dispersion of monocytes size.

Consensus definitions for sepsis 2016 include lactate $>2 \mathrm{mmol} / \mathrm{L}$ in the definition of septic shock. Bilirubin and creatinine are used to determine the progression of organ failure and are related to worse prognosis. The initial diagnostic protocols also include leukocyte count and differential, with attention to immature granulocytes. New assays in microbiology allow for rapid and specific identification of pathogens if sepsis is suspected (48). 
Different biomarkers have been proposed to diagnose sepsis or to predict mortality, CRP, PCT, presepsin, IL-6, lipopolysaccharide-binding protein (LBP), neutrophil CD64 (nCD64), soluble triggering receptor expressed on myeloid cells-1 (sTREM-1) and the serum soluble urokinase-type plasminogen activator receptor (suPAR), among others $(49,50)$. These new biomarkers are promising, but due to their lack of sensitivity and specificity cannot be considered as independent tests. Therefore, given the clinical variability and the possibility of false positive or false negative results, no diagnosis should be made on the basis of a single laboratory test result. All analytical data must be put in an adequate clinical context.

The CPD represents a promising set of data to be included in the assessment of patients at risk of sepsis. New technologies have provided better testing, it is important to evaluate the usefulness, performance and validity of these biomarkers before implementing them in routine clinical care (48). These new tests, could be incorporated into practice to improve care, translating the technology into better outcomes for patients (51).

\section{Conclusions}

Sepsis is a complex inflammatory syndrome, severe, high prevalent and one of the major causes of morbidity and mortality in critically ill patients. Early diagnosis and treatment intervention reduces mortality. For this purpose, combination of biomarkers is required to improve the patients outcomes. New Hematology parameters can play a role in the early diagnosis of sepsis with impact in clinical practice.

\section{Acknowledgments}

Funding: None.

\section{Footnote}

Conflicts of Interest: The author has completed the ICMJE uniform disclosure form (available at http://dx.doi. org/10.21037/atm-19-3173). The author has no conflicts of interest to declare.

Ethical Statement: The author is accountable for all aspects of the work in ensuring that questions related to the accuracy or integrity of any part of the work are appropriately investigated and resolved.
Open Access Statement: This is an Open Access article distributed in accordance with the Creative Commons Attribution-NonCommercial-NoDerivs 4.0 International License (CC BY-NC-ND 4.0), which permits the noncommercial replication and distribution of the article with the strict proviso that no changes or edits are made and the original work is properly cited (including links to both the formal publication through the relevant DOI and the license). See: https://creativecommons.org/licenses/by-nc-nd/4.0/.

\section{References}

1. Fleischmann C, Scherag A, Adhikari NKJ, et al. Assessment of global incidence and mortality of hospitaltreated sepsis: current estimates and limitations. Am J Respir Crit Care Med 2016;193:259-72.

2. Gyawali B, Ramakrishna K, Dhamoon AS. Sepsis: The evolution in definition, pathophysiology, and management. SAGE Open Med 2019;7:2050312119835043.

3. Levy MM, Artigas A, Phillips PS, et al. Outcomes of the Surviving Sepsis Campaign in intensive care units in the USA and Europe: a prospective cohort study. Lancet Infect Dis 2012;12:919-24.

4. Shashikumar SP, Stanley MD, Sadiq I, et al. Early sepsis detection in critical care patients using multiscale blood pressure and heart rate dynamics. J Electrocardiol 2017;50:739-43.

5. Martins PS, Kallas EG, Neto MC, et al. Upregulation of reactive oxygen species generation and phagocytosis and an increased apoptosis in human neutrophils during severe sepsis and septic shock. Shock 2003;20:208-12.

6. Hotchkiss RS, Karl IE. The pathophysiology and treatment of sepsis. N Engl J Med 2003;348:138-50.

7. Brown KA, Brain SD, Pearson JD. Neutrophils in development of multiple organ failure in sepsis. Lancet 2006;368:157-69.

8. Segal AW. How neutrophils kill microbes. Annu Rev Immunol 2005;23:197-223.

9. Tak T, van Groenendael R, Pickkers P, et al. Monocyte subsets are differentially lost from the circulation during acute inflammation induced by human experimental endotoxemia. J Innate Immun 2017;9:464-74.

10. Yona S, Jung S. Monocytes: subsets, origins, fates and functions. Curr Opin Hematol 2010;17:53-9.

11. Hoffmann JJML. New hematology analyzer parameters and their clinical relevance. EFLM Newsl 2018;1:3-5.

12. Green R. Development, History, and Future of Automated Cell Counters. Clin Lab Med 2015;35:1-10. 
13. Chabot-Richards DS, George TI. White blood cell counts: reference methodology. Clin Lab Med 2015;35:11-24.

14. Chaves F, Tierno B, Xu D. Quantitative determination of neutrophil VCS parameters by the Coulter automated hematology analyzer: new and reliable indicators for acute bacterial infection. Am J Clin Pathol 2005;124:440-4.

15. Xu D. Clinical Applications of Leukocyte Morphological Parameters. Int J Pathol Clin Res 2015;1:1-4.

16. Celik IH, Demirel G, Aksoy HT, et al. Automated determination of neutrophil VCS parameters in diagnosis and treatment efficacy of neonatal sepsis. Pediatr Res 2012;71:121-5.

17. Tang H, Xu D. Reference change values of leukocyte numerical and morphological parameters determined by Coulter DxH800. Int J Lab Hematol 2013;35:e24-6.

18. Jean A, Boutet C, Lenormand B, et al. The new haematology analyzer $\mathrm{DxH} 800$ : an evaluation of the analytical performances and leucocyte flags, comparison with the LH 755. Int J Lab Hematol 2011;33:138-45.

19. Park DH, Park K, Park J, et al. Screening of sepsis using leukocyte cell population data from the Coulter automatic blood cell analyzer DxH800. Int J Lab Hematol 2011;33:391-9.

20. Linssen J. Automation and Validation of a Rapid Method to Assess Neutrophil and Monocyte Activation by Routine Fluorescence Flow Cytometry In Vitro. Cytometry B Clin Cytom 2008;74:295-309.

21. Urrechaga E, Bóveda O, Aguirre U. Improvement in detecting sepsis using leukocyte cell population data (CPD). Clin Chem Lab Med 2019;57:918-26.

22. Urrechaga E, Bóveda O, Aguirre U. Neutrophil Cell Population Data biomarkers for Acute Bacterial Infection. J Pathol Infect Dis 2018;1:1-7.

23. Raimondi F, Ferrara T, Capasso L, et al. Automated determination of neutrophil volume as screening test for late-onset sepsis in very low birth infants. Pediatr Infect Dis J 2010;29:288

24. Lee JC, Ahern TP, Chaves FP, et al. Utility of hematologic and volume, conductivity, and scatter parameters from umbilical cord blood in predicting chorioamnionitis. Int J Lab Hematol 2010;32:351-9.

25. Celik IH, Demirel G, Sukhachev D, et al. Neutrophil volume, conductivity and scatter parameters with effective modeling of molecular activity statistical program gives better results in neonatal sepsis. Int J Lab Hematol 2013;35:82-7.

26. Cortegiani A, Di Benedetto A, Marino L, et al. Evaluation of automated hematologic VCS parameters in severe sepsis and septic shock: a case-control study. Crit Care 2010;14:P31.

27. Chaves F, Tierno B, Xu D. Neutrophil volume distribution width: a new automated hematologic parameter for acute infection. Arch Pathol Lab Med 2006;130:378-80.

28. Mardi D, Fwity B, Lobmann R, et al. Mean cell volume of neutrophils and monocytes compared with C-reactive protein, interleukin-6 and white blood cell count for prediction of sepsis and nonsystemic bacterial infections. Int J Lab Hematol 2010;32:410-8.

29. Lee AJ, Gyung Kim SG. Mean cell volumes of neutrophils and monocytes are promising markers of sepsis in elderly patients Blood Res 2013;48:193-7.

30. Mammen J, Choudhuri J, Paul J, et al. Cytomorphometric Neutrophil and Monocyte Markers May Strengthen the Diagnosis of Sepsis. J Intensive Care Med 2018;33:656-62.

31. Arora P, Gupta PK, Lingaiah R, et al. Volume, conductivity, and scatter parameters of leukocytes as early markers of sepsis and treatment respons. J Lab Physicians 2019;11:29-33.

32. Crouser ED, Parrillo JE, Seymour C, et al. Improved Early Detection of Sepsis in the ED With a Novel Monocyte Distribution Width Biomarker. Chest 2017;152:518-26.

33. Luo Y, Lin J, Chen H, et al. Utility of neut-X, neut-Y and neut- $Z$ parameters for rapidly assessing sepsis in tumor patients. Clin Chim Acta 2013;422:5-9.

34. Park SH, Park CJ, Lee BR, et al. Sepsis affects most routine and cell population data (CPD) obtained using the Sysmex XN-2000 blood cell analyzer: neutrophil-related CPD NE-SFL and NE-WY provide useful information for detecting sepsis. Int J Lab Hematol 2015;37:190-8.

35. Buoro S, Seghezzi M, Vavassori M, et al. Clinical significance of cell population data (CPD) on Sysmex XN9000 in septic patients with our without liver impairment. Ann Transl Med 2016;4:418.

36. Shalini P, Rao PS, Rao S. A Diagnostic Utility of Cell Population Data (CPD) In Sepsis Using Automated Hematology Analysers. APALM 2019;6:284-8.

37. Urrechaga E, Bóveda O, Aguirre U. Role of leucocytes cell population data in the early detection of sepsis. J Clin Pathol 2018;71:259-66.

38. van der Geest PJ, Mohseni M, Linssen J, et al. The intensive care infection score - a novel marker for the prediction of infection and its severity. Crit Care 2016;20:180-6.

39. Weimann K, Zimmermann M, Spies CD, et al. Intensive Care Infection Score--A new approach to distinguish between infectious and noninfectious processes in 
intensive care and medicosurgical patients. J Int Med Res 2015;43:435-51.

40. Tang H, Jing J, Bo D, et al. Biological variations of leukocyte numerical and morphologic parameters determined by UniCel DxH 800 hematology analyzer. Arch Pathol Lab Med 2012;136:1392-6.

41. Ricós C, Perich C, Minchinela J, et al. application of biological variation: a review. Biochem Medica 2009; 19:250-9.

42. Buoro S, Carobene A, Seghezzi M, et al. Short- and medium-term biological variation estimates of leukocytes extended to differential count and morphology-structural parameters (cell population data) in blood samples obtained from healthy people. Clin Chim Acta 2017;473:147-56.

43. Perez I, Redin E. Stability of leukocyte research parameters over time on the Sysmex XN: How to quantify the changes in cell morphology. Int J Lab Hem 2018. doi: 10.1111/ijlh.12866.

44. Seghezzi M, Buoro S, Previtali G, et al. A preliminary proposal for quality control assessment and harmonization of leukocytes morphology-structural

Cite this article as: Urrechaga E. Reviewing the value of leukocytes cell population data (CPD) in the management of sepsis. Ann Transl Med 2020;8(15):953. doi: 10.21037/atm-193173 parameters (cell population data parameters). J Med Biochem 2018;37:486-98.

45. Singer M, Deutschman CS, Seymour CW, et al. The third international consensus definitions for sepsis and septic shock (Sepsis-3). JAMA 2016;315:801-10.

46. Larsen FF, Petersen JA. Novel biomarkers for sepsis: A narrative review. Eur J Intern Med 2017;45:46-50.

47. Karon BS, Tolan NV, Wockenfus AM, et al. Evaluation of lactate, white blood cell count, neutrophil count, procalcitonin and immature granulocyte count as biomarkers for sepsis in emergency department patients. Clin Biochem 2017;50:956-8.

48. Fan SL, Miller NS, Lee J, et al. Diagnosing sepsis: the role of laboratory medicine, Clin Chim Acta 2016;460:203-10.

49. Faix JD. Biomarkers of sepsis. Crit Rev Clin Lab Sci 2013;50:23-36.

50. Lippi G. Sepsis biomarkers: past, present and future. Clin Chem Lab Med 2019;57:1281-3.

51. Graber ML, Patel M, Claypool S. Sepsis as a model for improving diagnosis. Diagnosis 2018;5:3-10. 